Relations industrielles

Industrial Relations

\title{
Les implications salariales des comportements de consommation
}

\section{Marc-Adélard Tremblay}

Volume 24, numéro 1, 1969

URI : https://id.erudit.org/iderudit/027986ar

DOI : https://doi.org/10.7202/027986ar

Aller au sommaire du numéro

Éditeur(s)

Département des relations industrielles de l'Université Laval

ISSN

0034-379X (imprimé)

1703-8138 (numérique)

Découvrir la revue

Citer cet article

Tremblay, M.-A. (1969). Les implications salariales des comportements de consommation. Relations industrielles / Industrial Relations, 24(1), 63-70. https://doi.org/10.7202/027986ar
Résumé de l'article

L'auteur tente ici de traduire la manière dont le consommateur se conçoit lui-même, essaie de faire ressortir les pressions qui s'exercent sur ce dernier et définit les ressources dont il devrait disposer pour se procurer les biens et services qu'il juge nécessaires
Tous droits réservés @ C Département des relations industrielles de l'Université Laval, 1969
Ce document est protégé par la loi sur le droit d'auteur. L’utilisation des services d'Érudit (y compris la reproduction) est assujettie à sa politique d'utilisation que vous pouvez consulter en ligne.

https://apropos.erudit.org/fr/usagers/politique-dutilisation/ 
On a tenté de ré-habiliter les repères (guidelines) américains en se demandant si l'augmentation des salaires (et des prix) n'aurait pas été plus importante sans eux. Tout ce qu'on a pu dire, c'est qu'ils «semblaient » avoir ralenti l'augmentation des salaires, mais qu'il était possible que d'autres facteurs en aient été la cause ${ }^{(10)}$.

\section{Conclusion}

Dans ces circonstances, comment expliquer la popularité des discussions sur une politique de salaires? Avec beaucoup de dureté, des auteurs ont affirmé que "when a large mass feels threatened by development over which it has little or no influence, it is likely to be easily manipulated and to be pleased by gestures that bring little tangible benefit but do serve as a reassurance ${ }^{(11)}$.

N'est-on pas alors en droit de se demander si les prises de position, les gestes et les discours sur la politique de salaires n'ont pas seulement pour but d'augmenter la liberté d'action, le statut ou le pouvoir politique de ceux qui en parlent?

\section{Les implications salariales des comportements de consommation}

\section{Marc-Adélard Tremblay}

L'auteur tente ici de traduire la manière dont le consommateur se conçoit lui-même, essaie de faire ressortir les pressions qui s'exercent sur ce dernier et définit les ressources dont il devrait disposer pour se procurer les biens et services qu'il juge nécessaires.

\section{Introduction}

Dans ce bref exposé, nous nous plaçons sous l'angle de l'indivi-
TREMBLAY, Marc-Adélard, Ph.D., professeur, Département de sociologie et d'anthropologie, Université Laval, Québec.

(10) G. L. Perry, «Wages and Guideposts », dans American Economic Review, sept. 1967 , p. 897 et sq.

(ii) M. Edelman et R. W. Fleming, op. cit., p. 309. 
du qui, disposant de certaines ressources financières, consomme des biens et des services. Nous nous efforcerons de traduire la manière dont il se conçoit en tant que consommateur, les pressions qui s'exercent sur lui pour l'amener effectivement à acheter certains biens et certains services et les ressources dont il devrait disposer pour s'approprier les biens et les services qu'il juge nécessaires.

Nous nous appuierons sur les résultats d'une étude ${ }^{(1)}$ sur les comportements de consommation de la famille salariée menée au Québec il y a dix ans (1958) pour dégager les grandes tendances de la consommation chez les travailleurs. Celles-ci nous amèneront à nous interroger sur leur signification vis-à-vis les politiques salariales en 1968.

\section{Les caractéristiques de la consommation}

L'ensemble des résultats de cette enquête conduite en collaboration avec mon collègue Fortin sont assez bien connus. Nous ne ferons que les rappeler très brièvement ici afin qu'elles puissent servir de tremplin à une analyse des répercussion qu'elles entraînent sur les politiques de maintien du revenu.

\section{DIVERSIFICATION DANS LA NATURE DES BESOINS}

À peu près $30 \%$ des revenus du travailleur servent à satisfaire des besoins nouveaux, tels que l'automobile, le mobilier moderne et: les appareils ménagers électriques, les loisirs commercialisés et les vacances ainsi que le coût des assurances.

\section{L'HOMOGÉNÉITÉ DES BESOINS}

Nous voulons dire par là que l'ensemble des consommateurs ont tendance à définir leurs besoins (les biens et services jugés nécessaires à leur bien-être) de la même manière peu importe qu'ils vivent à la ville ou à la campagne, qu'ils soient des manœuvres ou des cols blancs, qu'ils aient une faible scolarité ou des niveaux supérieurs d'instruction et qu'ils gagnent $\$ 3,000, \$ 6,000$, ou $\$ 8,000.00$ par année. Comprenez ici que les besoins sont un élément purement subjectif. L'homogénéité de cette attitude, découle à notre point de vue, de l'influence toute puissante des normes de consommation imposées par les communications de masse et la publicité commerciale.

(') Tremblay, M. A. et Fortin, G.: Les comportements économiques de la famille salariée du Québec, Presses de l'Université Laval, Québec, 1964, 405 pages. 
LE NIVEAU DE REVENU EST LE FACTEUR QUI DÉTERMINE LE NIVEAU OBJECTIF DE CONSOMMATION

Si la conception subjective de ce qui est nécessaire pour bien vivre est constamment extensible, l'aptitude qu'aura un individu à satisfaire ses besoins dépend, du niveau objectif (réel) de son revenu disponible. Ce niveau de consommation permet à l'individu soit d'être privé par rapport à des biens essentiels (niveau des privations réelles), soit de satisfaire tout juste ses besoins, (emprisonnement dans l'univers des besoins), soit encore de réaliser des projets (pénétration dans l'univers des aspirations).

LE MINIMUM DE BIEN-ÊTRE SE SITUE AUTOUR DE $\$ 4,500.00$

Les individus fixent à $\$ 4,500.00$ par année le niveau de revenu minimum capable d'assurer leur bien-être. C'est dire que ces revenus leur permettraient de consommer les biens essentiels. Sans avoir effectué d'enquête pour mettre à date ce chiffre, nous rehausserions aujourd'hui ce seuil à $\$ 5,500.00$. Cette nouvelle norme tient compte d'une double tendance qui s'est accentuée

a) la hausse du coût de la vie,

b) l'élévation du niveau des besoins et des aspirations.

\section{LE RECOURS AU CRÉdit EST DE PLUS EN PLUS GÉNÉRALISÉ}

La publicité commerciale devance le consommateur en fabriquant un modèle de consommateur moyen dont les capacités effectives dépassent celles du salarié moyen. Les biens ainsi proposés dans ses messages sont perçus comme des nécessités de la vie quotidienne. Le recours au crédit devient alors le moyen le plus courant pour satisfaire ses besoins, aidant les uns à alléger leurs privations mais plaçant les autres dans une situation financière encore plus précaire à la suite des nouvelles obligations qu'ils viennent de contracter. La majorité des usagers du crédit ne finissent jamais de payer leurs dettes.

\section{L'ÉPARGNE EST NÉCESSAIRE : SEULS LES PLUS FAVORISÉS PEUVENT ÉPARGNER}

Si la totalité des travailleurs définissent l'épargne comme nécessaire pour garantir la sécurité et assurer les « vieux jours 》 c'est une minorité de privilégiés qui peuvent effectivement épargner dans le sens traditionnel du terme. 


\section{Les politiques salariales}

Quelles exigences peut-on dégager de ces observations malheureusement simplifiées et fragmentaires sous l'angle des politiques salariales? Poser le problème dans ces termes nous oblige à élargir les cadres de la discussion et à nous référer à des ordres de phénomène beaucoup plus vastes que la simple politique des salaires. Les commentaires que nous voulons effectuer se regroupent autour de trois thèmes, soit: 1. Un niveau de vie convenable. 2. Une économie progressive. 3. Une politique de sécurité sociale. Nous les discuterons dans cet ordre.

\section{UN NIVEAU DE VIE CONVENABLE}

Par rapport à nos sociétés techniques et modernes, il ne saurait être question comme nous l'avons signalé d'assurer seulement à l'ensemble des individus un minimum vital. Il faut dépasser ce seuil des nécessités matérielles les plus essentielles pour garantir à l'individu un optimum de bien-être. D'ailleurs dans ce type de société les gouvernements se préoccupent d'alléger la pauvreté des pays les moins biens favorisés.

En dépit de cette idéologie de la société opulente, une «troisième solitude» de plus en plus nombreuse se développe dans notre société en marge des groupes qui bénéficient de la richesse. Bien loin d'avoir atteint le niveau de bien-être auquel nous faisions allusion, ils sont privés des biens les plus essentiels. L'existence des «économiquement faibles 》 des pauvres et des indigents dans notre société est un fait brutal qui témoigne de nos inaptitudes.

L'optimum de bien-être doit se définir par rapport à un optimum de besoins, celui-ci devant être relativement stable. J'admets qu'il est extrêmement difficile de fixer arbitrairement une norme précise. Mais il est un fait inéluctable : le niveau des besoins des salariés s'élève à un rythme plus rapide que le niveau de leurs disponibilités financières. Sans vouloir déprécier les gains salariaux des travailleurs des récentes années, (ils étaient mérités) n'y a-t-il point lieu d'envisager une stabilisation des niveaux de salaire, des contrôles plus sévères sur la nature et le contenu de la publicité commerciale afin d'atténuer la psychologie de la consommation massive, et d'éduquer le consommateur sur ses capacités réelles (et non fictives) de consommation? La stabilité des salaires, le freinage des aspirations ainsi que l'apprentissäge à une saine administration financière m'apparaissent comme étant certaines techniques - parmi d'autres - pour empêchev la croissance de la pauvreté et faciliter l'accession à un niveau optimum de bien-être. 


\section{UNE ÉCONOMIE PROGRESSIVE}

Les objectifs énoncés par le Conseil économique du Canada pour favoriser l'expansion et la croissance économique sont trop bien connus pour les répéter ici. Nous allons plutôt faire allusion à quelques uns des éléments économiques qui influent directement sur le niveau des salaires, et, par voie de conséquence, sur les niveaux de consommation, à savoir : le plein emploi, le maintien du pouvoir d'achat et la promotion professionnelle des travailleurs.

Il semble très difficile, dans notre système économique d'atteindre le plein emploi et d'éviter le chômage des travailleurs à certaines périodes de l'année. Dans l'étude à laquelle nous faisions allusion, au début, les niveaux de vie insatisfaisants des travailleurs étaient dûs soit à des salaires réguliers trop bas, ou soit encore à des salaires irréguliers. Les conséquences sociales du chômage sont désastreuses pour les individus et les familles.

Le maintien du pouvoir d'achat est une autre condition économique pré-requise à la recherche individuelle du bien-être.

Finalement, la préparation professionnelle du travailleur, sa compétence technique, sa motivation au travail, ses possibilités de recyclage et d'avancement, sa productivité sont autant de conditions essentielles à une politique générale de promotion des travailleurs.

\section{VERS UNE POLITIQUE INTÉGRÉE DES DIFFÉRENTES MESURES DE BIEN-ÊTRE}

Par mesures de bien-être, nous entendons un ensemble de circonstances objectives qui possèdent un rendement économique et social. Les structures existent, les individus les connaissent et s'en servent; elles lui procurent des biens et des services qui entraînent des niveaux de satisfaction et des niveaux correspondants de bien-être. Le but ultime d'une politique globale et intégrée de sécurité sociale, c'est de libérer les individus complètement de leurs privations, c'est de leur permettre de jouir de niveaux de vie qui rendent possible les aspirations; c'est 'de leur donner l'ensemble des garanties qui les prémunissent contre l'ensemble des risques inhérents à la vie.

\section{Libérer l'individu de ses privations}

La plupart du temps cette fin s'exprime d'une manière positive. Il s'agit de permettre à l'individu de satisfaire ses besoins les plus essentiels. 
Ces besoins (en aliments, en vêtements, en logement, en chauffage, en soins médicaux, etc.) peuvent être satisfaits à la condition que l'individu jouisse d'un minimum vital. Cette conception est dépassée dans ce sens que le minimum vital peut rarement permettre à l'individu d'atteindre un niveau « acceptable » de bien-être. C'était là d'ailleurs exprimer une définition limitative du besoin. Mais si on élargit la notion de besoin à l'univers complet des besoins à satisfaire, cela devient un objectif irréalisable par le seul biais de la sécurité sociale.

Réduire les privations de l'individu apparaît un objectif de portée plus réaliste. Il s'agit alors d'élaborer une politique qui vise à assister ceux qui, laissés à eux-mêmes, ne peuvent se procurer les biens et les services nécessaires pour mener une vie convenable. Du même coup, on réduit les inégalités économiques, on allège les charges financières trop lourdes d'individus défavorisés, on réduit les sentiments individuels de privation et de frustation.

\section{Permettre à l'individu de franchir le seuil de l'univers des aspirations}

(Un niveau de vie convenable)

La deuxième fin intermédiaire possède elle aussi un fondement économique. Elle vise directement le rehaussement du niveau de vie des individus afin qu'ils puissent non seulement satisfaire l'ensemble de leurs besoins mais également nourrir des projets. N'étant plus enfermé dans l'univers de leurs besoins, ils peuvent songer à l'avenir, entretenir des aspirations. Voilà que nous touchons plus concrètement à un aspect dynamique de la sécurité sociale. Au lieu d'être uniquement un ensemble de mesures palliatives qui atténuent les difficultés, nous visons ici directement des mesures de libération individuelle et de promotion sociale. On sait que les individus qui ont des préoccupations par rapport à la satisfaction de leurs besoins sont rivés au quotidien et incapables de faire des plans et de prévoir l'avenir. Ce sentiment de prévoyance à long terme diminue substantiellement l'insécurité de l'individu.

\section{Prémunir l'individu contre un ensemble de risques.}

La notion de risque introduit l'élément hasard: tous les individus sont susceptibles d'être frappés à un moment donné. Si les risques sont universels (accidents, maladies coûteuses, vieillesse, infirmité et incapacité, perte d'emploi, etc.) certaines catégories d'individus sont plus susceptibles que d'autres d'être frappés. Par exemple, ceux qui vivent dans des milieux économiquement faibles, qui ont des bas niveaux d'instruction 
et qui n'ont pas de métier, sont plus susceptibles que d'autres d'être en chômage et d'être privés de revenus réguliers. Cette notion de risque est si bien connue et elle s'identifie tellement à la conception traditionnelle de la sécurité sociale qu'il n'est point nécessaire d'insister.

La finalité de la sécurité sociale est de produire le bien-être des individus non pas seulement dans l'immédiat mais aussi avec continuité. Elle découle d'une philosophie de la vie en société : dignité et épanouissement de la personne, responsabilités individuelles et collectives, progrès économique et social sont les fondements même de cette conception de la vie sociale. Cette philosophie s'incarne dans des types d'organisation sociale et se traduit dans des techniques qui visent à les maintenir.

\section{Conclusion}

La sécurité sociale concerne finalement le bien-être individuel puisque seuls les individus sont capables de ressentir la satisfaction que procure le sentiment de bien-être. La sécurité sociale est essentiellement une action coopérative de la collectivité vis-à-vis ses membres considérés individuellement. La sécurité sociale est née des contraintes d'une organisation sociale qui limite le pouvoir de satisfaction des besoins individuels à la capacité de travail et à la capacité de gain. Elle est née de l'inégalité économique dont on s'attachait d'abord à corriger les plus graves conséquences pour évoluer peu à peu d'une notion de minimum tolérable à la notion d'un optimum de bien-être. Traditionnellement, divers champs d'action parallèles se sont imposés :

a) des programmes visant à fournir le revenu de remplacement soit sous forme d'assistance financière, soit sous forme d'allocations à des fins de consommation spécifiques. Ceci lorsque les circonstances privent l'individu de sa capacité de gagner (invalidité, chômage, vieillesse), ou lorsque les charges familiales accentuent l'écart entre les niveaux de ressources et de besoins.

b) des programmes de prévoyance qui offrent à l'individu une protection contre les risques sociaux. C'est toute la gamme des assurances sociales auxquelles sont attachées des prestations en espèces ou en nature.

c ) des programmes visant à diminuer l'ampleur des risques sociaux par des groupes d'individus ou pour l'ensemble de la population par le truchement des politiques de recyclage et de prolongement de la scolarité. 
L'ensemble de ces programmes sociaux ont comme objet principal soit la réduction des privations de l'individu et de sa famille, soit la réduction de l'insécurité économique et sociale. Ils pourront avoir comme effet de situer l'individu dans un «univers d'aspirations légitimes > à l'intérieur duquel se situe l'optimum de bien-être. Le niveau optimum de bien-être sera fonction en dernier ressort du niveau des ressources disponibles et de certains choix quant à l'allocation de ces ressources. Dans l'optique d'une optimisation de la fonction de bien-être, l'allocation devra répondre aux ordres de préférence individuels et collectifs.

\section{Conclusion générale}

Il faudrait, en dernier ressort, discuter des exigences théoriques d'une politique globale, cohérente de sécurité sociale, de la nature de l'intervention étatique dans ce domaine et des freins structurels qui ralentissent son action.

\section{La politique salariale et le cycle économique}

\section{Yves Dubé}

Dans cet exposé, l'auteur explique comment il ne croit pas plus à la politique salariale qu'il peut croire à l'inévitabilité du cycle économique. Il croit encore moins à la possibilité d'influencer le cycle économique par une quelconque politique salariale.

Le titre de ce travail semble suggérer qu'il doit y avoir une politique salariale et que celle-ci doit être définie en tenant compte du cycle économique. C'est ce que plusieurs prétendent. Mais notre thèse sera tout autre. En fait, nous ne croyons pas plus à la politique salariale que nous pouvons croire à l'inévitabilité du cycle économique. Nous croyons encore moins à la possibilité d'influencer le cycle économique par une quelconque politique salariale.

Pour démontrer notre thèse nous tenterons d'examiner ce qu'a été au cours de la présente décen-

DUBE, Yves, doyen, Faculté des sciences sociales, professeur, Département d'économique, Université Laval, QUEBEC. 